\title{
Chiral transition in a strong magnetic background
}

\author{
Eduardo S. FragA* and Ana Júlia Mizher ${ }^{\dagger}$ \\ Instituto de Física, Universidade Federal do Rio de Janeiro, \\ Caixa Postal 68528, Rio de Janeiro, RJ 21941-972, Brazil
}

\begin{abstract}
The presence of a strong magnetic background can modify the nature and the dynamics of the chiral phase transition at finite temperature. We compute the modified effective potential in the linear sigma model with quarks to one loop in the $\overline{M S}$ scheme for $N_{f}=2$. For fields $e B \sim 5 m_{\pi}^{2}$ and larger a crossover is turned into a weak first-order transition. We discuss possible implications for non-central heavy ion collisions at RHIC and LHC, and for the primordial QCD transition.
\end{abstract}

\section{INTRODUCTION}

Strong magnetic fields can produce remarkable physical effects. One of the most spectacular examples is provided by magnetars [1]. More recently, it has been proposed that strong magnetic fields could play a very important role in the physics of high-energy heavy ion collisions, affecting observables in the case of non-central collisions at the Relativistic Heavy Ion Collider (RHIC), at Brookhaven, and the Large Hadron Collider (LHC), at CERN, and providing a possible signature of the presence of CP-odd domains in the presumably formed quarkgluon plasma (QGP) phase [2]. In that case, one would reach magnetic fields $B \sim 10^{19}$ Gauss, which correspond to $e B \sim 6 m_{\pi}^{2}$, where $e$ is the fundamental charge and $m_{\pi}$ is the pion mass. This gives a very intense magnetic background from the point of view of quantum chromodynamics (QCD) scales [4].

In this paper we investigate the effects of a strong magnetic background on the nature and dynamics of the chiral phase transition at finite temperature, $T$, and vanishing chemical potential. As a framework, we adopt the linear sigma model coupled to quarks $\left(\operatorname{LSM}_{q}\right)$ with two flavors, $N_{f}=2$ [3]. This effective theory has been widely used to describe different aspects of the chiral transition, such as thermodynamic properties $[4,5,6,7,8,19,10,11,12,13,14]$ and the nonequilibrium phase conversion process [15], as well as combined to other models in order to include effects from confinement [16, 17].

In the limit of strong magnetic fields, we find that the nature of the chiral transition is modified. The original $\operatorname{LSM}_{q}$, with no magnetic field, yields a crossover at $T=T_{c} \sim 150 \mathrm{MeV}$ for a choice of coupling to quarks that provides a reasonable mass for the constituent quarks at zero temperature [8]. The presence of a strong magnetic background turns this picture into a weak firstorder phase transition. As a consequence, the dynamics that follows a rapid supercooling, such as the one that presumably happens in a high-energy heavy ion collision, can be dramatically modified due to the presence of a

\footnotetext{
*fraga@if.ufrj.br

†anajulia@if.ufrj.br
}

barrier in the effective potential. Even if the barrier is quite small, as in the case of a weak first-order transition, its effect can be very significant for the dynamics, holding the system in the false vacuum until it reaches the spinodal instability and explodes [18].

From the theoretical point of view, the non-trivial role played by magnetic fields in the nature of phase transitions has been known for a long time [19]. Modifications in the vacuum of quantum electrodynamics (QED) and QCD have also been investigated within different frameworks, mainly using effective models $20,21,22,23,24$, 25, 26], especially the NJL model [27], and chiral perturbation theory [28, 29, 30], but also resorting to the quark model [31] and certain limits of QCD [32]. Most treatments have been concerned with vacuum modifications by the magnetic field, though medium effects were considered in a few cases. More recently, interesting phases in dense systems [33], as well as effects on the dynamical quark mass [34] and on the quark-hadron transition 35] were also considered.

The paper is organized as follows. Section II presents briefly the low-energy effective model adopted in this paper, as well as a discussion of the approximations required. In Section III we show our results for the modified effective potential, in the presence of a strong magnetic background. Phenomenological consequences are illustrated and discussed in Section IV. Section V contains our conclusions and outlook.

\section{EFFECTIVE THEORY}

To describe the chiral phase structure of strong interactions at finite temperature we adopt the $\mathrm{LSM}_{q}$, defined by the following lagrangian

$$
\begin{aligned}
\mathcal{L} & =\bar{\psi}_{f}\left[i \gamma^{\mu} \partial_{\mu}-g\left(\sigma+i \gamma_{5} \vec{\tau} \cdot \vec{\pi}\right)\right] \psi_{f} \\
& +\frac{1}{2}\left(\partial_{\mu} \sigma \partial^{\mu} \sigma+\partial_{\mu} \vec{\pi} \partial^{\mu} \vec{\pi}\right)-V(\sigma, \vec{\pi})
\end{aligned}
$$

where

$$
V(\sigma, \vec{\pi})=\frac{\lambda}{4}\left(\sigma^{2}+\vec{\pi}^{2}-v^{2}\right)^{2}-h \sigma
$$

is the self-interaction potential for the mesons, exhibiting both spontaneous and explicit breaking of chiral symmetry. The $N_{f}=2$ massive fermion fields $\psi_{f}$ represent the 
up and down constituent-quark fields $\psi=(u, d)$. The scalar field $\sigma$ plays the role of an approximate order parameter for the chiral transition, being an exact order parameter for massless quarks and pions. The latter are represented by the pseudoscalar field $\vec{\pi}=\left(\pi^{0}, \pi^{+}, \pi^{-}\right)$, and it is common to group together these meson fields into an $O(4)$ chiral field $\phi=(\sigma, \vec{\pi})$.

In what follows, we implement a simple mean-field treatment with the customary simplifying assumptions (see, e.g., Ref. [8]). Quarks constitute a thermalized fluid that provides a background in which the long wavelength modes of the chiral condensate evolve. Hence, at $T=0$, the model reproduces results from the usual LSM without quarks or from chiral perturbation theory for the broken phase vacuum [36]. In this phase, quark degrees of freedom are absent (excited only for $T>0$ ). The $\sigma$ field is heavy, $M_{\sigma} \sim 600 \mathrm{MeV}$, and treated classically. On the other hand, pions are light, and fluctuations in $\pi^{+}$and $\pi^{-}$couple to the magnetic field, $B$, as will be discussed in the next section, whereas fluctuations in $\pi^{0}$ give a $B$ independent contribution that we ignore, for simplicity. For $T>0$, quarks are relevant (fast) degrees of freedom and chiral symmetry is approximately restored in the plasma for high enough $T$. In this case, we incorporate quark thermal fluctuations in the effective potential for $\sigma$, i.e. we integrate over quarks to one loop. Pions become rapidly heavy only after $T_{c}$ and their fluctuations can, in principle, matter since they couple to $B$. However, they will play a minor role in this case, as will be clear later, so that we ignore their thermal fluctuations in this section, for simplicity.

The parameters of the lagrangian are chosen such that the effective model reproduces correctly the phenomenology of QCD at low energies and in the vacuum, in the absence of a magnetic field, such as the spontaneous (and small explicit) breaking of chiral symmetry and experimentally measured meson masses. So, one has to impose that the chiral $S U_{L}(2) \otimes S U_{R}(2)$ symmetry is spontaneously broken in the vacuum, and the expectation values of the condensates are given by $\langle\sigma\rangle=f_{\pi}$ and $\langle\vec{\pi}\rangle=0$, where $f_{\pi}=93 \mathrm{MeV}$ is the pion decay constant. The explicit symmetry breaking term is determined by the PCAC relation which gives $h=f_{\pi} m_{\pi}^{2}$, where $m_{\pi} \approx 138 \mathrm{MeV}$ is the pion mass. This yields $v^{2}=f_{\pi}^{2}-m_{\pi}^{2} / \lambda$. The value of $\lambda=20$ leads to a $\sigma$ mass, $m_{\sigma}^{2}=2 \lambda f_{\pi}^{2}+m_{\pi}^{2}$, equal to $600 \mathrm{MeV}$. In mean field theory, the purely bosonic part of this Lagrangian exhibits a second-order phase transition [37] at $T_{c}=\sqrt{2} v$ if the explicit symmetry breaking term, $h_{q}$, is dropped. For $h_{q} \neq 0$, the transition becomes a smooth crossover from the restored to broken symmetry phases. For $g>0$, one has to include a finite-temperature one-loop contribution from the quark fermionic determinant to the effective potential. The choice $g=3.3$, which yields a reasonable mass for the constituent quarks in the vacuum, $M_{q}=g f_{\pi}$, leads to a crossover at finite temperature and vanishing chemical potential, and will be adopted in this paper [42].
Standard integration over the fermionic degrees of freedom [36], using a classical approximation for the chiral field, gives the following formal expression for the effective potential in the $\sigma$ direction:

$$
V_{e f f}(T, \sigma)=V(\sigma)-\frac{T}{\mathcal{V}} \ln \operatorname{det}\left[\frac{\left(G_{E}^{-1}+M_{q}(\sigma)\right)}{T}\right],
$$

where $V(\sigma)$ is the classical self-interaction potential for the mesonic sector in the $\sigma$ direction, $G_{E}$ is the fermionic Euclidean propagator, $M_{q}$ is the effective fermion mass in the presence of the chiral field background, and $\mathcal{V}$ is the volume of the system. To one loop, one has $V_{\text {eff }}=$ $V(\phi)+V_{q}(\phi)$, where the contribution from the quarks reads

$$
V_{q} \equiv-\nu_{q} T \int \frac{d^{3} k}{(2 \pi)^{3}} \ln \left(1+e^{-E_{k}(\sigma) / T}\right) .
$$

where $\nu_{q}=24$ is the color-spin-isospin-baryon charge degeneracy factor, $E_{k}(\sigma)=\left(\vec{k}^{2}+M_{q}^{2}(\sigma)\right)^{1 / 2}$, and $M(\sigma)=$ $g|\sigma|$ plays the role of an effective mass for the quarks. The net effect of this term is correcting the potential for the chiral field, approximately restoring chiral symmetry for a critical temperature $T_{c} \sim 150 \mathrm{MeV}[8]$. The effective potential $V_{\text {eff }}$ for several values of the temperature is displayed in Fig. 1, assuming $g=3.3$.

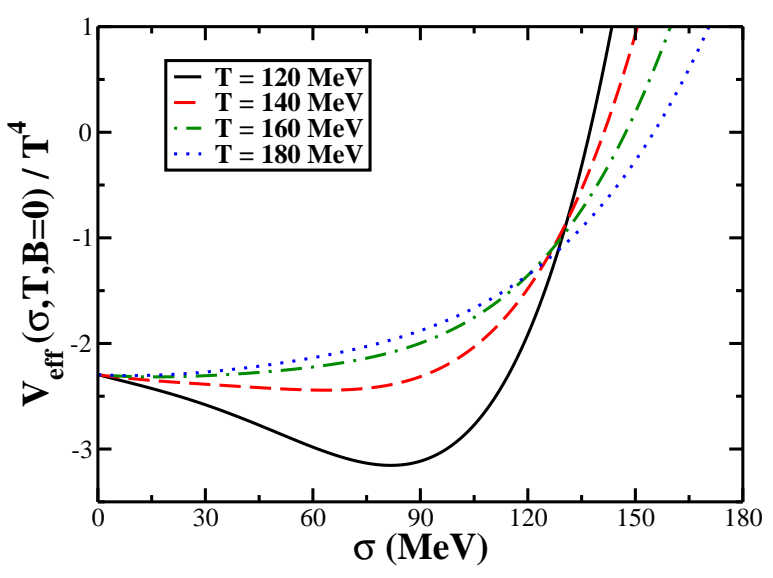

FIG. 1: $V_{\text {eff }}(\sigma)$ for different values of the temperature for $g=3.3$, in the absence of a magnetic field.

\section{MODIFIED EFFECTIVE POTENTIAL}

Let us now assume that the system is in the presence of a strong magnetic field background that is constant and homogeneous. For definiteness, let us take the direction of the magnetic field as the $z$-direction, $\vec{B}=B \hat{z}$. One can compute the modified effective potential by redefining the dispersion relations of the scalar and spinor fields in the presence of $\vec{B}$, using the minimal coupling shift in 
the gradient and the field equations of motion. For this purpose, it is convenient to choose the gauge such that $A^{\mu}=\left(A^{0}, \vec{A}\right)=(0,-B y, 0,0)$.

For scalar fields with electric charge $q$, one has

$$
\begin{aligned}
& \left(\partial^{2}+m^{2}\right) \phi=0, \\
& \partial_{\mu} \rightarrow \partial_{\mu}+i q A_{\mu} .
\end{aligned}
$$

After decomposing $\phi$ into Fourier modes, except for the dependence in the coordinate $y$, one obtains

$$
\begin{aligned}
\varphi^{\prime \prime}(y) & +2 m\left[\left(\frac{p_{0}^{2}-p_{z}^{2}-m^{2}}{2 m}\right)\right. \\
& \left.-\frac{q^{2} B^{2}}{2 m}\left(y+\frac{p_{x}}{q B}\right)^{2}\right] \varphi(y)=0,
\end{aligned}
$$

which has the form of a Schrödinger equation for a harmonic oscillator. Its eigenmodes correspond to the wellknown Landau levels

$$
\varepsilon_{n} \equiv\left(\frac{p_{0 n}^{2}-p_{z}^{2}-m^{2}}{2 m}\right)=\left(n+\frac{1}{2}\right) \omega_{B}
$$

where $\omega_{B}=|q| B / m$ and $n$ is an integer, and provide the new dispersion relation:

$$
p_{0 n}^{2}=p_{z}^{2}+m^{2}+(2 n+1)|q| B
$$

One can proceed in an analogous way for fermions with charge $q$. From the free Dirac equation $\left(i \gamma^{\mu} \partial_{\mu}-m\right) \psi=$ 0 , and the shift in $\partial_{\mu}$, one arrives at the following Schrödinger equation

$$
\begin{aligned}
u_{\sigma}^{\prime \prime}(y) & +2 m\left[\left(\frac{p_{0}^{2}-p_{z}^{2}-m^{2}+q B \sigma}{2 m}\right)\right. \\
& \left.-\frac{q^{2} B^{2}}{2 m}\left(y+\frac{p_{x}}{q B}\right)^{2}\right] u_{\sigma}(y)=0
\end{aligned}
$$

which yields the new dispersion relation for quarks:

$$
p_{0 n}^{2}=p_{z}^{2}+m^{2}+(2 n+1-\sigma)|q| B .
$$

It is also straightforward to show that integrals over four momenta and thermal sum-integrals acquire the following forms, respectively:

$$
\begin{aligned}
& \int \frac{d^{4} k}{(2 \pi)^{4}} \mapsto \frac{|q| B}{2 \pi} \sum_{n=0}^{\infty} \int \frac{d k_{0}}{2 \pi} \frac{d k_{z}}{2 \pi}, \\
& T \sum_{\ell} \int \frac{d^{3} k}{(2 \pi)^{3}} \mapsto \frac{|q| B T}{2 \pi} \sum_{\ell} \sum_{n=0}^{\infty} \int \frac{d k_{z}}{2 \pi},
\end{aligned}
$$

where $n$ represents the different Landau levels and $\ell$ stands for the Matsubara frequency indices [36].

According to the assumptions of our effective model, the vacuum potential will be modified by a contribution from the charged pions that couple to the magnetic field. To one loop, this is given by the following integral:

$$
V_{\pi^{ \pm}}^{V}=\frac{1}{2} \frac{|q| B}{2 \pi} \sum_{n=0}^{\infty} \int_{-\infty}^{+\infty} \frac{d k}{2 \pi}\left[k^{2}+m_{\pi}^{2}+(2 n+1)|q| B\right]^{1 / 2}
$$

For sufficiently high magnetic fields, $e B>>m_{\pi}^{2}$, and ignoring contributions independent of the chiral condensate, this simplifies to the following renormalized result [43]:

$$
V_{\pi^{+}}^{V}+V_{\pi^{+}}^{V}=-\frac{2 m_{\pi}^{2} e B}{32 \pi^{2}} \log 2
$$

where we have added the contributions from $\pi^{+}$and $\pi^{-}$.

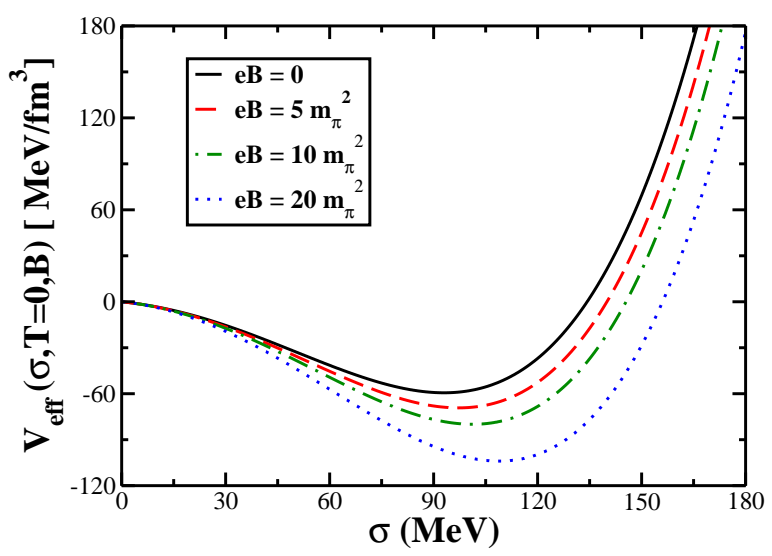

FIG. 2: $V_{\text {eff }}(\sigma, T, B)$ for $T=0$ and different values of the magnetic field.

Results for the modified vacuum potential are displayed in Fig. 2 for different values of the background magnetic field. As one can see from the plot, the presence of the magnetic field enhances the value of the chiral condensate and the depth of the broken phase minimum of the modified effective potential, a result that is in line with those found within different approaches (see, for instance, Refs. [26, 28, 30]). This could be an indication that the presence of a strong magnetic background might favor a weak first-order transition over the crossover that is obtained for $B=0$. To investigate this point, one has to compute the modified thermal contributions to the effective potential and study the process of chiral symmetry restoration.

Charged pions contribute to the one-loop thermal corrections via

$$
V_{\pi^{ \pm}}^{T}=\frac{|q| B T}{2 \pi} \sum_{n=0}^{\infty} \int_{-\infty}^{+\infty} \frac{d k}{2 \pi} \log \left(1-e^{-\beta \omega_{\pi n}}\right),
$$

whereas the contribution from quarks reads 


$$
V_{q}^{T}=-\sum_{\sigma= \pm} \frac{|q| B T}{2 \pi} \sum_{n=0}^{\infty} \int_{-\infty}^{+\infty} \frac{d k}{2 \pi} 2 \log \left(1+e^{-\beta \omega_{n \sigma}}\right)
$$

where, for quarks, one still has to sum over colors, which brings a factor $N_{c}$, and over flavors. The latter sum, differently from what happened in the previous section, does not bring and overall $N_{f}$ factor, since the electric charges are not equal, and their absolute values add up to one. In the presence of a nonzero $B$, spins are also treated separately, as is clear in the expression above.

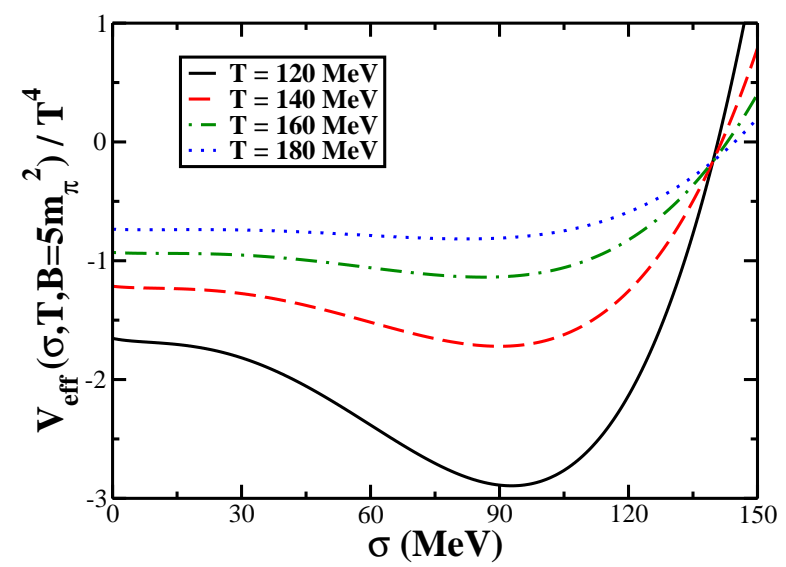

FIG. 3: $V_{e f f}(\sigma, T, B)$ for $e B=5 m_{\pi}^{2}$ and different values of the temperature.

For sufficiently high magnetic fields, not only compared to the pion mass but also to the effective quark mass and to the temperature, and again ignoring contributions independent of the chiral condensate, we obtain for charged pions and quarks, respectively:

$$
V_{\pi^{+}}^{T}+V_{\pi^{-}}^{T}=-2 \frac{(e B)^{2}}{(2 \pi)^{3 / 2}}\left(\frac{T^{2}}{e B}\right)^{3 / 4} e^{-\beta \sqrt{e B}}\left[1+\frac{m_{\pi}^{2}}{4 e B}\right]
$$

$$
\begin{aligned}
V_{q}^{T} & =-N_{c} \frac{e B T^{2}}{2 \pi^{2}}\left[\int_{-\infty}^{+\infty} d x \log \left(1+e^{-\sqrt{x^{2}+m_{f}^{2} / T^{2}}}\right)\right. \\
& \left.+\sqrt{2 \pi \beta \sqrt{2 e B}} e^{-\beta \sqrt{2 e B}}\left(1+\frac{m_{f}^{2}}{8 e B}\right)\right]
\end{aligned}
$$

One can notice that the pion thermal contribution and part of the quark thermal contribution are exponentially suppressed for high magnetic fields, which essentially comes from an increase in the effective mass due to the magnetic field $\sim e^{-m_{e f f} / T} \sim e^{-\# \sqrt{e B} / T}$. Therefore, within these approximations, the total modified effective

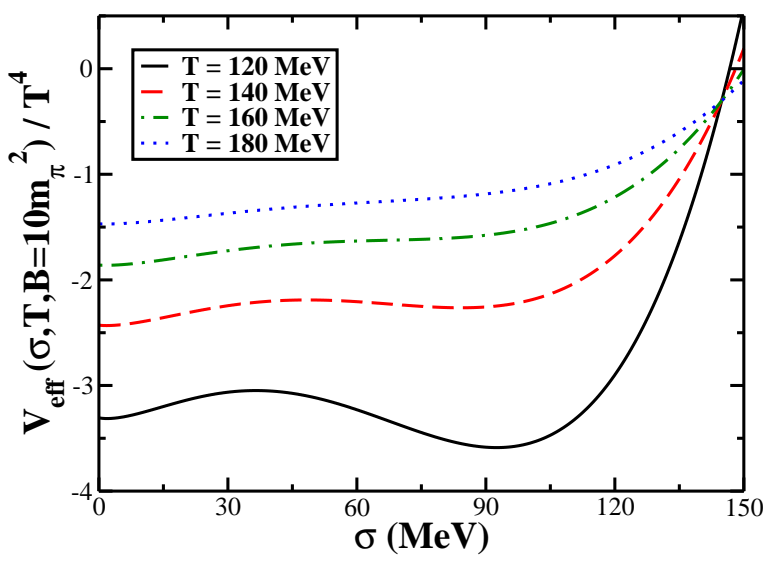

FIG. 4: $V_{\text {eff }}(\sigma, T, B)$ for $e B=10 m_{\pi}^{2}$ and different values of the temperature.

potential is given by

$$
\begin{gathered}
V_{\text {eff }}(\sigma, T, B) \approx \frac{\lambda}{4}\left(\sigma^{2}-v^{2}\right)^{2}-h \sigma-2 \frac{m_{\pi}^{2} e B}{32 \pi^{2}} \log 2 \\
-2 N_{c} \frac{e B T^{2}}{2 \pi^{2}} \int_{0}^{\infty} d x \log \left(1+e^{-\sqrt{x^{2}+m_{f}^{2} / T^{2}}}\right)
\end{gathered}
$$

The modified effective potential, $V_{e f f}(\sigma, T, B)$, is plotted for different temperatures for $e B=5 m_{\pi}^{2}, e B=10 m_{\pi}^{2}$ and $e B=20 m_{\pi}^{2}$ in Fig. 3, Fig. 4 and Fig. 5, respectively. For $e B=5 m_{\pi}^{2}$, one verifies that the critical temperature becomes higher, $T_{c}>200 \mathrm{MeV}$. Moreover, a closer look uncovers the presence of a tiny barrier, signaling a first-order phase transition. A zoom for a couple of temperatures close to $T_{c}$ is shown in Fig. 6 . In the case of higher magnetic fields, the existence of the barrier is rather clear, showing that the presence of a strong magnetic background can really modify the nature of the chiral transition, in this case from a crossover to a firstorder transition whose strength depends on how intense $B$ is. In Figs. 4, 5 and 6, we considered the same set of temperatures to allow for a more direct comparison.

Another important feature that comes out of this comparison is the fact that for $e B=10 m_{\pi}^{2}$ the critical temperature drops again $\left(T_{c}<140 \mathrm{MeV}\right)$ as compared to the case in which $e B=5 m_{\pi}^{2}$. This phenomenon continues to happen for higher $B$ (see Fig. 6), showing that there is a non-trivial balance between temperature and magnetic field that can bring some richness to the chiral $T-B$ phase diagram that should be explored. The presence of the barrier is not qualitatively modified, though, and the "conversion" to a first-order transition seems to be robust. 


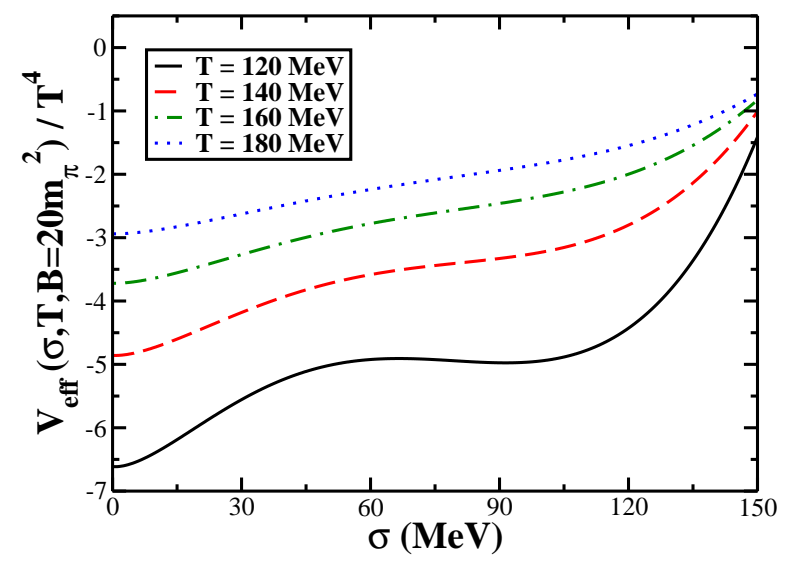

FIG. 5: $V_{\text {eff }}(\sigma, T, B)$ for $e B=20 m_{\pi}^{2}$ and different values of thr

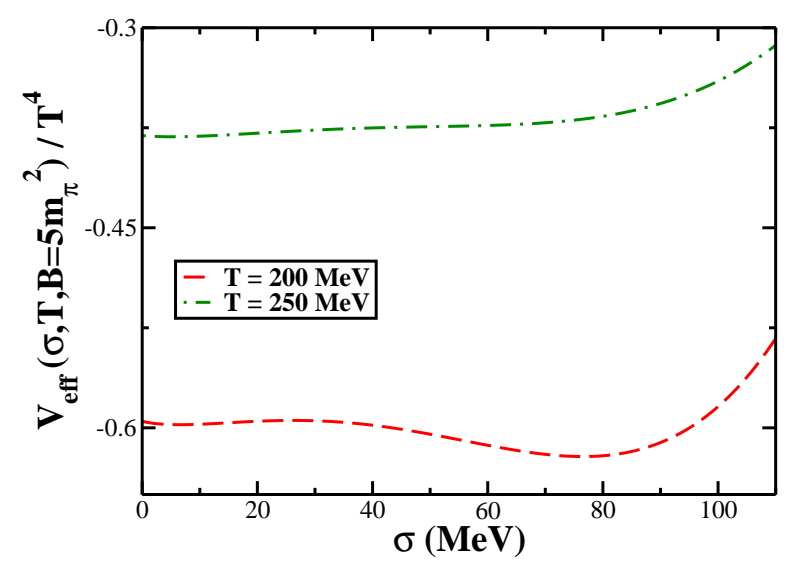

FIG. 6: $V_{e f f}(\sigma, T, B)$ for $e B=5 m_{\pi}^{2}$ and different values of the temperature (zoom near the critical temperature to exhibit the barrier).

\section{PHENOMENOLOGICAL CONSEQUENCES}

Besides the pure theoretical interest in the modification of the nature of the chiral phase transition by the presence of a strong magnetic background, one should consider possible phenomenological implications within the reach of present experiments. At RHIC, estimates by Kharzeev, McLerran and Warringa [2] give $e B \sim 5.3 m_{\pi}^{2}$. For the LHC, there is a factor $Z_{P b} / Z_{A u}=82 / 79$ coming from the difference in charge, and some small increase in the maximum value of $e B$ due to the higher center-ofmass energy (as observed for RHIC [2]). So, it is reasonable to consider $e B \sim 6 m_{\pi}^{2}$ as a representative estimate.

We show in Fig. 7 a zoom of the effective potential for $e B \sim 6 m_{\pi}^{2}$ for a temperature slightly below the critical one, whereas the general picture is not appreciably different from the case $e B \sim 5 m_{\pi}^{2}$, and can be seen in Fig. 3 . Comparing Figs. 1 and 3, one can establish the basic differences brought about a magnetic field of the magnitude that could possibly be found in non-central high-energy heavy ion collisions. One moves from a crossover sce- nario to that of a weak first-order chiral transition, with a critical temperature $\sim 30 \%$ higher.

In the case of a crossover, one expects the system to be smoothly drained to the true vacuum, with no formation of bubbles or spinodal decomposition. In the case of the first order transition depicted in Fig. 3, despite the fact that the barrier is quite small, part of the system will be kept in the false vacuum for a while, a few bubbles will be formed, depending on the intensity of the supercooling, and spinodal instability will then set in [18].

The case of the $\mathrm{LSM}_{q}$ with a coupling constant $g=5.5$ exhibits a first-order phase transition even for $B=0$, and has been often used to study nucleation and spinodal decomposition phenomena in the chiral transition 6, 9, 10, 12, 14, 15, 17]. There, one finds a barrier $\sim 0.25$ in the effective potential for temperatures close to $T_{c}$, in units of $T^{4}$, and it has been shown that the system stays mostly apprisionated in the false vacuum until it reaches the spinodal explosive phase conversion regime [9]. For $g=3.3$, we saw that there is a very weak first-order chiral transition for $B>0$, with a barrier an order of magnitude smaller (see Fig. 7). Nevertheless, even such small barriers can hold the system in the false vacuum until the spinodal instability in the case of a fast supercooling, as expected for high-energy heavy ion collisions [18]. Therefore, the existence of a strong magnetic background can bring significant effects to the dynamics of the chiral transition.

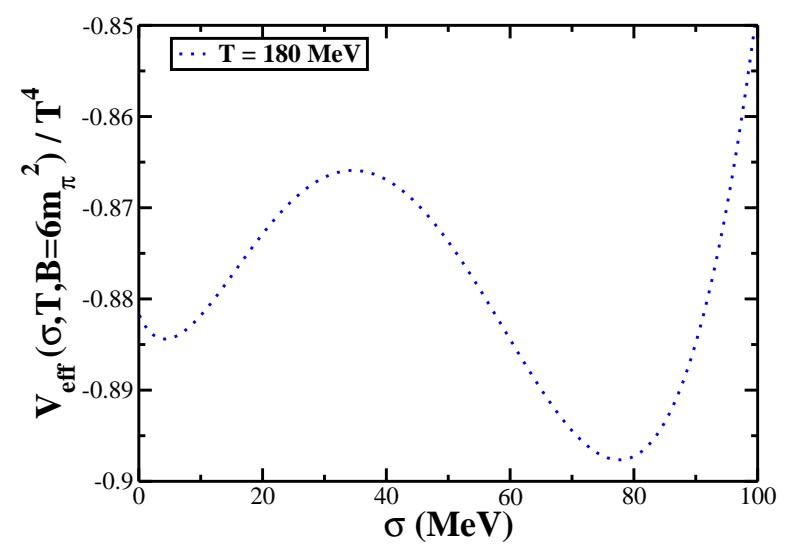

FIG. 7: $V_{e f f}(\sigma, T, B)$ for $e B=6 m_{\pi}^{2}$ for $T=180 \mathrm{MeV}$.

\section{CONCLUSIONS AND OUTLOOK}

Lattice QCD indicates a crossover instead of a firstorder chiral transition at finite temperature and vanishing chemical potential. However, a strong magnetic background might modify this situation. Our computation of the modified effective potential clearly shows the formation of a barrier that seems to be robust, in the case of magnetic fields that are larger than the other relevant 
scales, namely the pion mass and the temperature. On the other hand, it is clear that further investigation of the low magnetic field regime at finite temperature, for both $B<T$ and $B \sim T$, in the context of the chiral transition is called for, since the phase diagram seems to exhibit non-trivial features in this region of parameters which is interesting for critical phenomena in strong interactions.

For heavy ion collisions at RHIC and LHC, the barrier in the effective potential seems to be quite small. Nevertheless, it can probably hold most of the system in a metastable state down to the spinodal explosion, implying a different dynamics of phase conversion as compared to the crossover scenario. Moreover, the phenomenology resulting from varying $T$ and $B$ seems to be rich: there seems to be a competition between strengthening the chiral symmetry breaking via vacuum effects and its restoration by the thermal (magnetic) bath. In particular, non-central heavy ion collisions might show features of a first-order transition when contrasted to central collisions.

The dynamics of the chiral phase transition will certainly be affected by the presence of a strong magnetic background, since the nature of the transition is modified. In order to study its impact on the relevant time scales of this process, one has to perform real-time simulations of the evolution of the order parameter in the presence of the modified effective potential, e.g., in a Langevin framework. Results in this direction will be presented elsewhere [38].

Of course, the description presented in this paper is admittedly very simple. In actual heavy ion collisions, the magnetic field is neither uniform nor constant in time, and the values quoted here represent estimates of its maximum magnitude. In fact, the field intensity rapidly decreases with time, and any of the effects discussed here should manifest in the early-time dynamics. Moreover, if there is a a fast variation of $B$ with time, there will be an induced electric field that could break the vacuum through the Schwinger mechanism of pair production, and this would modify the condensate [30] and the vacuum effective potential. Therefore a more realistic description of the external electromagnetic field effects on the chiral transition must be pursued before one is able to make explicit phenomenological predictions. On the other hand, we believe that the results presented here, even if seen as just qualitative, are encouraging. In particular, the possibility that strong magnetic fields can turn a crossover into a first-order transition might be relevant for the physics of the primordial QCD transition, eventually reconciling lattice results that predict a crossover [39] to the description of phase conversion via bubble nucleation that assume a first-order phase transition [40].

\section{Acknowledgments}

We thank J.-B. Blaizot and A. Starinets for discussions. The authors would also like to thank E. Iancu for his kind hospitality at the Service de Physique Théorique, CEA/Saclay, where this work has been concluded. This work was partially supported by CAPES-COFECUB, CNPq, FAPERJ and FUJB/UFRJ.
[1] R. C. Duncan and C. Thompson, Astrophys. J. 392, L9 (1992); C. Thompson and R. C. Duncan, ibid. 408, 194 (1993).

[2] I. V. Selyuzhenkov [STAR Collaboration], Rom. Rep. Phys. 58, 049 (2006); D. Kharzeev, Phys. Lett. B 633, 260 (2006); D. E. Kharzeev, L. D. McLerran and H. J. Warringa, arXiv:0711.0950 [hep-ph].

[3] M. Gell-Mann and M. Levy, Nuovo Cim. 16, 705 (1960).

[4] L. P. Csernai and I. N. Mishustin, Phys. Rev. Lett. 74, 5005 (1995); A. Abada and J. Aichelin, Phys. Rev. Lett. 74, 3130 (1995); A. Abada and M. C. Birse, Phys. Rev. D 55, 6887 (1997);

[5] I. N. Mishustin and O. Scavenius, Phys. Rev. Lett. 83, 3134 (1999).

[6] O. Scavenius and A. Dumitru, Phys. Rev. Lett. 83, 4697 (1999).

[7] H. C. G. Caldas, A. L. Mota and M. C. Nemes, Phys. Rev. D 63, 056011 (2001).

[8] O. Scavenius, A. Mocsy, I. N. Mishustin and D. H. Rischke, Phys. Rev. C 64, 045202 (2001).

[9] O. Scavenius, A. Dumitru, E. S. Fraga, J. T. Lenaghan and A. D. Jackson, Phys. Rev. D 63, 116003 (2001).

[10] K. Paech, H. Stoecker and A. Dumitru, Phys. Rev. C 68, 044907 (2003); K. Paech and A. Dumitru, Phys. Lett. B 623, 200 (2005).

[11] A. Mocsy, I. N. Mishustin and P. J. Ellis, Phys. Rev. C
70, 015204 (2004).

[12] C. E. Aguiar, E. S. Fraga and T. Kodama, J. Phys. G 32, 179 (2006).

[13] B. J. Schaefer and J. Wambach, Phys. Rev. D 75, 085015 (2007).

[14] B. G. Taketani and E. S. Fraga, Phys. Rev. D 74, 085013 (2006).

[15] E. S. Fraga and G. Krein, Phys. Lett. B 614, 181 (2005).

[16] R. D. Pisarski, Phys. Rev. D 62, 111501 (2000); A. Dumitru and R. D. Pisarski, Phys. Lett. B 504, 282 (2001); A. Dumitru and R. D. Pisarski, Nucl. Phys. A 698, 444 (2002).

[17] O. Scavenius, A. Dumitru and A. D. Jackson, Phys. Rev. Lett. 87, 182302 (2001).

[18] J. D. Gunton, M. San Miguel and P. S. Sahni, in Phase Transitions and Critical Phenomena (Eds.: C. Domb and J. L. Lebowitz, Academic Press, London, 1983), v. 8.

[19] L. D. Landau and E. M. Lifshitz, Statistical Physics Course of Theoretical Physics, volume 5 (ButterworthHeinemann, 1984).

[20] S. P. Klevansky and R. H. Lemmer, Phys. Rev. D 39, 3478 (1989).

[21] V. P. Gusynin, V. A. Miransky and I. A. Shovkovy, Phys. Lett. B 349, 477 (1995); V. P. Gusynin, V. A. Miransky and I. A. Shovkovy, Nucl. Phys. B 462, 249 (1996).

[22] A. Y. Babansky, E. V. Gorbar and G. V. Shchepanyuk, 
Phys. Lett. B 419, 272 (1998).

[23] K. G. Klimenko, arXiv:hep-ph/9809218

[24] G. W. Semenoff, I. A. Shovkovy and L. C. R. Wijewardhana, Phys. Rev. D 60, 105024 (1999).

[25] A. Goyal and M. Dahiya, Phys. Rev. D 62, 025022 (2000).

[26] B. Hiller, A. A. Osipov, A. H. Blin and J. da Providencia, SIGMA 4, 024 (2008).

[27] S. P. Klevansky, Rev. Mod. Phys. 64, 649 (1992).

[28] I. A. Shushpanov and A. V. Smilga, Phys. Lett. B 402, 351 (1997).

[29] N. O. Agasian and I. A. Shushpanov, Phys. Lett. B 472, 143 (2000).

[30] T. D. Cohen, D. A. McGady and E. S. Werbos, Phys. Rev. C 76, 055201 (2007).

[31] D. Kabat, K. M. Lee and E. Weinberg, Phys. Rev. D 66, 014004 (2002).

[32] V. A. Miransky and I. A. Shovkovy, Phys. Rev. D 66, 045006 (2002).

[33] D. T. Son and M. A. Stephanov, Phys. Rev. D 77, 014021 (2008).

[34] K. G. Klimenko and V. C. Zhukovsky, arXiv:0803.2191 [hep-ph].

[35] N. O. Agasian and S. M. Fedorov, arXiv:0803.3156 [hep$\mathrm{ph}]$.

[36] J. I. Kapusta and C. Gale, Finite-Temperature Field Theory: Principles and Applications (Cambridge University Press, 2006).

[37] R. D. Pisarski and F. Wilczek, Phys. Rev. D 29, 338 (1984).

[38] A. J. Mizher and E. S. Fraga, to appear.

[39] E. Laermann and O. Philipsen, Ann. Rev. Nucl. Part. Sci. 53 (2003) 163.

[40] D. J. Schwarz, Annalen Phys. 12, 220 (2003).

[41] For comparison, one has $m_{\pi} / e \sim 10^{19}$ Gauss.

[42] It is also common to use $g=5.5$ when one aims to describe a first-order chiral transition. However, since the constituent quark mass is given by $M_{q}=g\langle\sigma\rangle$, this choice yields a constituent quark mass that is too high.

[43] High magnetic fields allow for an expansion of generalized zeta functions, of the form $\zeta\left(-1+\frac{\epsilon}{2}, \frac{m_{\pi}^{2}+|q| B}{2|q| B}\right)$, that appear in the $\overline{M S}$ computation of the momentum integral. 